\title{
mHealth and Health Information Technology Tools for Diverse Patients with Diabetes
}

\author{
Courtney R. Lyles, ${ }^{1}$ Neda Ratanawongsa, ${ }^{1}$ Shari D. Bolen, ${ }^{2}$ and Lipika Samal ${ }^{3}$ \\ ${ }^{1}$ University of California, San Francisco, CA, USA \\ ${ }^{2}$ Case Western Reserve University, Cleveland, $\mathrm{OH}, \mathrm{USA}$ \\ ${ }^{3}$ Harvard Medical School, Boston, MA, USA \\ Correspondence should be addressed to Courtney R. Lyles; courtney.lyles@ucsf.edu
}

Received 5 February 2017; Accepted 5 February 2017; Published 23 February 2017

Copyright (c) 2017 Courtney R. Lyles et al. This is an open access article distributed under the Creative Commons Attribution License, which permits unrestricted use, distribution, and reproduction in any medium, provided the original work is properly cited.

Mobile health (mHealth) and health information technology (HIT) tools to enhance diabetes health and healthcare management have proliferated rapidly, including websites, mobile phone applications, texting or interactive voice response phone calls, remote monitoring devices/sensors, and personal health records (PHRs) linked to electronic health records $[1,2]$. Many studies and systematic reviews have demonstrated that the additional communication and support provided by such technologies can improve outcomes like patient confidence, self-management, quality of life, and even health outcomes like glycemic control [3-10].

However, emerging evidence reveals a digital divide in health technology use, with lower use of widely disseminated technologies among racial/ethnic minority groups or those who have limited health literacy [11-14]. Although overall ownership and use of devices are increasing among $\mathrm{racial} / \mathrm{ethnic}$ minorities, lower income individuals, and other subgroups $[15,16]$, there remain access, skills, and interest barriers that influence this overall digital divide [17-19]. Furthermore, research in mHealth or HIT has not often directly engaged diverse end users, as evidenced by few published studies which report that the usability of diabetes technologies among participants represents the spectrum of technological proficiency or income and educational attainment [20].

This special issue therefore provides crucial evidence about the design, testing, and implementation of mHealth or health information technology platforms for diverse patients with diabetes. The included studies cover a range of relevant research on these topics.

Two studies describe HIT-facilitated interventions to enhance diabetes self-management support by engaging both patients and their families. L. S. Mayberry et al. describe an approach to user-centered design and iterative usability testing with low-income patients to develop an mHealth intervention to promote family engagement in self-management support. Although nontechnology facilitators may be needed to engage social support networks in diabetes self-care for this population, their findings suggest preliminary feasibility for low-income patients to engage in this text messaging self-management support. Meanwhile, A. H. Lansing et al. describe the feasibility of engaging rural teens with poorly controlled type 1 diabetes and their families through an Internet-delivered intervention to improve blood glucose self-monitoring and glycemic control. Their findings prompt future research directions about sustainability and scalability, particularly to teens and families who may not be as easily incentivized in similar web-delivered self-management programs.

Two studies describe novel approaches to tailoring mHealth interventions for culture and language. V. Fontil et al. describe an approach to leveraging academic-industry partnership in developing a culturally and linguistically appropriate diabetes prevention program tailored to safety net patients with limited health literacy. While their findings are limited to a small sample drawn from an academically 
affiliated clinic, the approach suggests a model for engaging health technology companies in designing products that will decrease the digital divide. P. Athavale et al. describe a health coaching intervention facilitated by automated telemedicine outreach for reducing diabetes among postpartum Latina women. They address the advantages and limitations of using HIT to improve the scalability of health coaching through community organizations like local Women, Infants, and Children (WIC) Programs in an effort to reach vulnerable women at high risk of loss to follow-up.

Finally, two papers describe future directions for research in the design and implementation of online patient portals and electronic health record systems (EHRs). D. Schillinger et al. describe a research protocol to partner with computational linguistics experts to study the linguistic complexity of secure messages between diverse patients with diabetes and their healthcare teams. They propose using this novel approach to quantify and study health literacy at a population level, while also developing tools to help care teams tailor their secure message content. N. Ratanawongsa et al. describe vulnerabilities in the ways EHR electronic prescribing has affected diabetes care for diverse patients and advocate for specific changes in EHR design, implementation, policy, and research.

It is well known that many existing technological interventions have not seen wide uptake among heterogeneous settings and patient populations [21] and multifaceted, realworld research strategies that can create insights for meaningful change in the near future. Overall, we believe this special issue offers innovative approaches for including diverse populations in mHealth and health technology research and inspires future informatics, implementation, and policy researchers to build on this important work. Notably, no interventions focused on using aggregated data from mobile technology or social media to design interventions. For instance, use of aggregated data on opportunities for healthy food and safe places to be active could be used to design future public health interventions to improve the built environment. Moving forward, we must continue these multiple strands of research to truly advance the field: from discovery of new technology programs that impact health behaviors, to adaptation of existing technologies for diverse user needs, to careful consideration of implementation strategies that might differentially impact patient subgroups. Findings from these studies also indicate that policy work around increasing and sustaining low-cost broadband access will also be critical to the future success of interventions to improve care and reduce disparities using HIT.

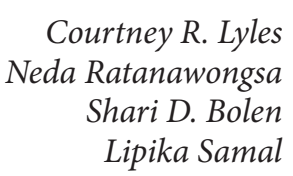

\section{References}

[1] S. R. Steinhubl, E. D. Muse, and E. J. Topol, "Can mobile health technologies transform health care?" JAMA, vol. 310, no. 22, pp. 2395-2396, 2013.
[2] R. Hillestad, J. Bigelow, A. Bower et al., "Can electronic medical record systems transform health care? Potential health benefits, savings, and costs," Health Affairs, vol. 24, no. 5, pp. 1103-1117, 2005.

[3] P. C. Tang and D. Lansky, "The missing link: bridging the patient-provider health information gap," Health Affairs, vol. 24, no. 5, pp. 1290-1295, 2005.

[4] K. Pal, S. V. Eastwood, S. Michie et al., "Computer-based interventions to improve self-management in adults with type 2 diabetes: a systematic review and meta-analysis," Diabetes Care, vol. 37, no. 6, pp. 1759-1766, 2014.

[5] J. D. Ralston, I. B. Hirsch, J. Hoath, M. Mullen, A. Cheadle, and H. I. Goldberg, "Web-based collaborative care for type 2 diabetes: a pilot randomized trial," Diabetes Care, vol. 32, no. 2, pp. 234-239, 2009.

[6] D. Schillinger, F. Wang, M. Handley, and H. Hammer, "Effects of self-management support on structure, process, and outcomes among vulnerable patients with diabetes: a three-arm practical clinical trial," Diabetes Care, vol. 32, no. 4, pp. 559-566, 2009.

[7] A. K. Hall, H. Cole-Lewis, and J. M. Bernhardt, "Mobile text messaging for health: a systematic review of reviews," Annual Review of Public Health, vol. 36, pp. 393-415, 2015.

[8] S. Hamine, E. Gerth-Guyette, D. Faulx, B. B. Green, and A. S. Ginsburg, "Impact of mHealth chronic disease management on treatment adherence and patient outcomes: a systematic review," Journal of Medical Internet Research, vol. 17, no. 2, 2015.

[9] B. M. Costa, K. J. Fitzgerald, K. M. Jones, and T. Dunning Am, "Effectiveness of IT-based diabetes management interventions: a review of the literature," BMC Family Practice, vol. 10, article no. 72, 2009.

[10] C. Y. Osborn, L. S. Mayberry, S. A. Mulvaney, and R. Hess, "Patient web portals to improve diabetes outcomes: a systematic review," Current Diabetes Reports, vol. 10, no. 6, pp. 422-435, 2010.

[11] U. Sarkar, A. J. Karter, J. Y. Liu et al., “The literacy divide: health literacy and the use of an internet-based patient portal in an integrated health system-results from the diabetes study of Northern California (DISTANCE)," Journal of Health Communication, vol. 15, no. 2, pp. 183-196, 2010.

[12] U. Sarkar, A. J. Karter, J. Y. Liu et al., "Social disparities in internet patient portal use in diabetes: evidence that the digital divide extends beyond access," Journal of the American Medical Informatics Association, vol. 18, no. 3, pp. 318-321, 2011.

[13] C. K. Yamin, S. Emani, D. H. Williams et al., "The digital divide in adoption and use of a personal health record," Archives of Internal Medicine, vol. 171, no. 6, pp. 568-574, 2011.

[14] T. Isaacs, D. Hunt, D. Ward, L. Rooshenas, and L. Edwards, “The inclusion of ethnic minority patients and the role of language in telehealth trials for type 2 diabetes: a systematic review," Journal of Medical Internet Research, vol. 18, no. 9, Article ID e256, 2016.

[15] Pew Internet \& American Life Project, Digital Differences, 2012, http://pewinternet.org/Reports/2012/Digital-differences.aspx.

[16] Pew Research Internet Project, Internet User Demographics: January 2014, 2014, http://www.pewinternet.org/data-trend/ internet-use/latest-stats/.

[17] K. Mossberger, C. J. Tolbert, D. Bowen, and B. Jimenez, "Unraveling different barriers to internet use: urban residents and neighborhood effects," Urban Affairs Review, vol. 48, no. 6, pp. 771-810, 2012.

[18] R. F. McCloud, C. A. Okechukwu, G. Sorensen, and K. Viswanath, "Beyond access: barriers to internet health information 
seeking among the urban poor," JAMA, vol. 23, no. 6, pp. 10531059, 2016.

[19] Pew Research Internet Project, Technology Adoption by Lower Income Populations, 2013, http://www.pewinternet.org/2013/ 10/08/technology-adoption-by-lower-income-populations/.

[20] C. R. Lyles, U. Sarkar, and C. Y. Osborn, "Getting a technologybased diabetes intervention ready for prime time: a review of usability testing studies," Current diabetes reports, vol. 14, no. 10, article no. 534, 2014.

[21] S. Kumar, W. J. Nilsen, A. Abernethy et al., "Mobile health technology evaluation: the mHealth evidence workshop," American Journal of Preventive Medicine, vol. 45, no. 2, pp. 228-236, 2013. 


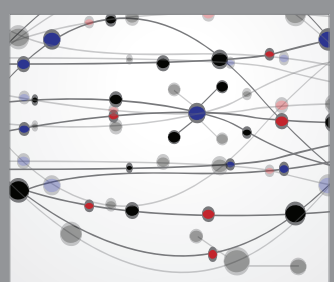

The Scientific World Journal
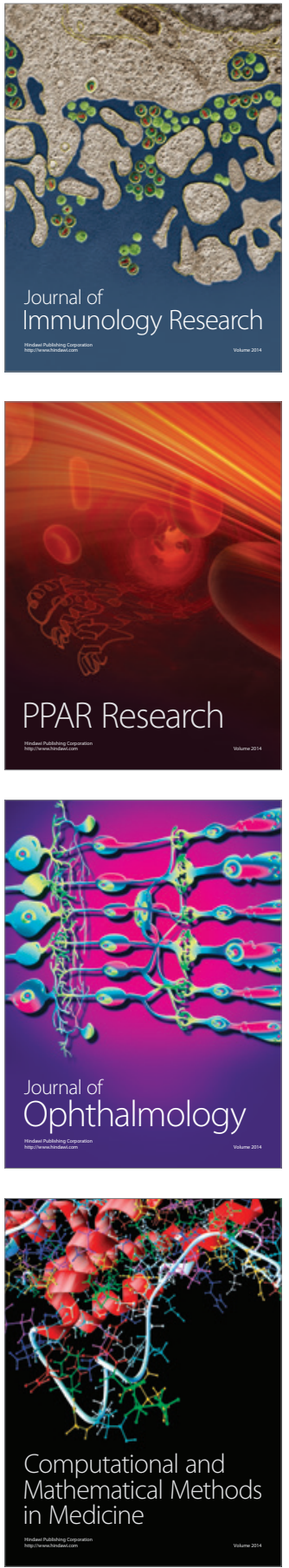

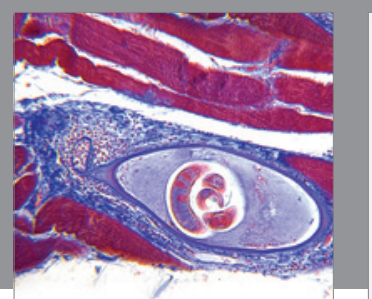

Gastroenterology Research and Practice
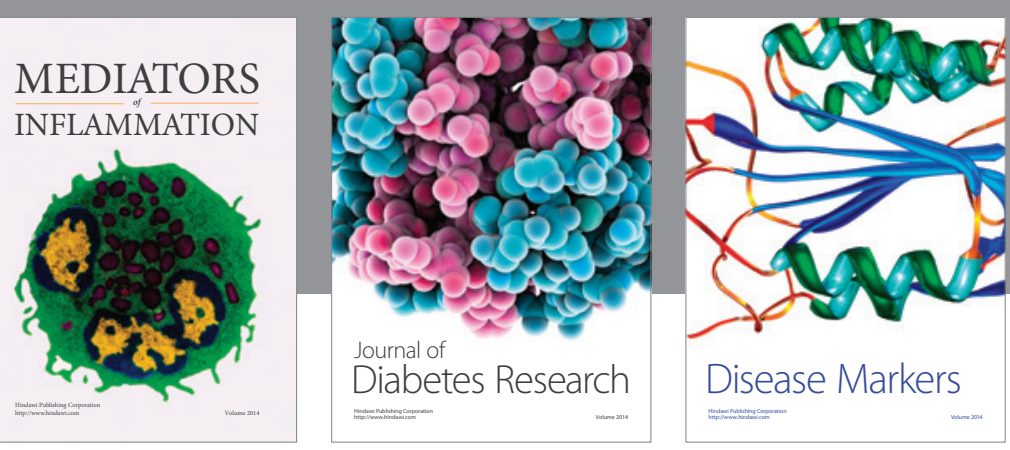

Disease Markers

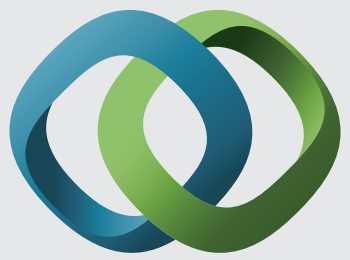

\section{Hindawi}

Submit your manuscripts at

https://www.hindawi.com
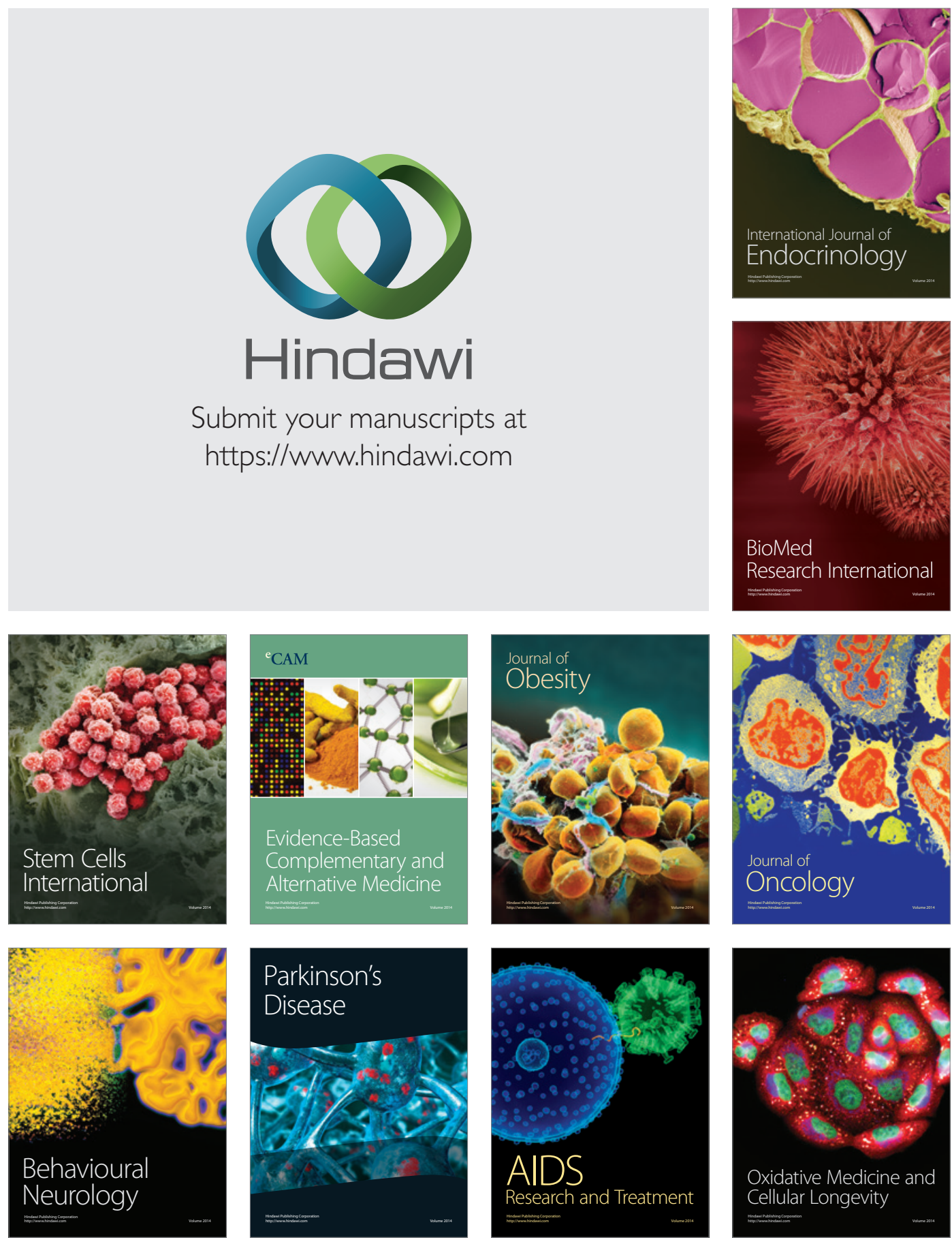